\title{
DETERMINANTES DE LA INNOVACIÓN: EVIDENCIA EN EL SECTOR MANUFACTURERO DE BOGOTÁ*
}

\author{
Esteban Álvarez** \\ William García ${ }^{* * *}$
}

Recibido: marzo 07 de 2011 - Aceptado: noviembre 13 de 2012

\section{RESUMEN}

El objetivo del presente artículo es analizar los determinantes de la innovación en el sector manufacturero de Bogotá a partir de diversos planteamientos teóricos y los resultados de un modelo de Tobit generalizado que tiene en cuenta las características estadísticas de los datos. Se encuentra que el tamaño de la firma, el capital extranjero, la capacitación de la mano de obra y el acceso a financiamiento son los factores que más influyen sobre la actividad innovadora en el caso analizado.

\section{PALABRAS CLAVE}

Innovación, sector manufacturero, Bogotá (Colombia), modelo Tobit generalizado.

\section{CLASIFICACIÓN JEL}

C34, L60, O12, O31

\section{CONTENIDO}

Introducción; 1. Teoría de la innovación; 2. Trabajos empíricos sobre innovación: revisión de la literatura; 3. Modelo econométrico; 4. Análisis de resultados; 5. Conclusiones y recomendaciones; Bibliografía; Anexos

\footnotetext{
Este artículo de investigación se deriva del trabajo de Grado para optar al título de Economista realizado por ambos autores bajo la supervisión del profesor Rafael Mora Castro y presentado a la Facultad de Administración y Economía de la Universidad Colegio Mayor de Cundinamarca, Bogotá. El proyecto comenzó en marzo 2010 - y finalizó en noviembre 2010. Al DANE, al DNP y a COLCIENCIAS, se les agradece el suministro de la información empleada en la elaboración del estudio. Los posibles errores presentes en el documento son completamente responsabilidad nuestra.

** Economista, Universidad Colegio Mayor de Cundinamarca, Bogotá, Colombia. Dirección. KR 114D\#147A17-201, Tel. (57-1) 6874913, E-mail esteban-al@hotmail.com.

*** Economista, Universidad Colegio Mayor de Cundinamarca, Bogotá. Especialista en Estadística, Universidad Nacional de Colombia, Bogotá. Asistente de Investigación, Latin America Human Mortality Database, Universidad Nacional de Colombia-CEDEPLAR, Belo Horizonte. Dirección. CL 71ª95-16, Tel. (57-1) 4341683, E-mail. wrgarciag@unal.edu.co.
} 


\section{INNOVATION DETERMINANTS: EVIDENCE IN THE MANUFACTURING SECTOR IN BOGOTA}

ABSTRACT

The objective of this article is to analyze the innovation determinants in the manufacturing sector in the city of Bogota parting from different theoretical statements and results drawn form a generalized Tobit model that considers the statistical characteristics of data. It is found that the size of the firm, foreign capital, labor training and access to financing are the factors that influence the most the innovative activity in the analyzed case.

\section{KEY WORDS}

Innovation, manufacturing sector, Bogota (Colombia) Tobit Generalized model.

\section{JEL CLASSIFICATION}

C34, L60, O12, O31

\section{CONTENT}

Introduction; 1. Innovation theory; 2. Empiric works over innovation: literature review; 3. Econometric model; 4. Result analysis; 5. Conclusions; Bibliography; Attachments

\section{DETERMINANTES DA INNOVAÇÃO: EVIDENCIA NO SETOR MANUFATUREIRO EM BOGOTÁ}

\section{RESUMO}

O objetivo do presente artigo é analisar os determinantes da inovação no setor manufatureiro em Bogotá a partir de diversos enfoques teóricos e os resultados do modelo de Tobit generalizado que tem em conta as caraterísticas estatísticas dos dados. Encontrou-se que o tamanho da firma, o capital estrangeiro, a capacitação da mão de obra e o acesso ao financiamento são os fatores que mais influem sobre a atividade inovadora no caso analisado.

\section{PALAVRAS CHAVE} Inovação, Setor de Manufatureiro, Bogotá (Colômbia), modelo Tobit generalizado.

\section{CLASIFICAÇÃO JEL}

C34, L60, O12, 031

\section{CONTEUDO}

Introdução; 1. Teoria da inovação; 2. Trabalhos empíricos sobre inovação: revisão da literatura; 3. Modelo Econométrico; 4. Analise de Resultados; 5. Conclusões; Bibliografia; Anexos 
Determinantes de la innovación: evidencia en el sector manufacturero de Bogotá

\section{INTRODUCCIÓN}

En la economía mundial la innovación es reconocida como uno de los factores clave de crecimiento económico y competitividad, por lo que es cada vez mayor el interés académico por comprender los factores que afectan la innovación en los países en desarrollo que presentan unas características distintivas con respecto a las evidenciadas en los países desarrollados. De esta manera resulta importante presentar evidencia robusta sobre los factores que impulsan o dificultan la actividad innovadora en el ámbito empresarial en estas economías.

Por consiguiente, el presente trabajo tiene como objetivo identificar el rol de algunos factores reconocidos como determinantes de la actividad innovadora, y cuantificar su impacto sobre los indicadores de innovación del sector manufacturero bogotano'; para ello, en primer lugar, se hace una revisión detallada de los principales trabajos que abordan el tema de manera teórica y empírica para establecer las hipótesis que guían el análisis, y en segundo lugar, se especifica un modelo Tobit generalizado estimado por máxima verosimilitud.

La metodología es acorde con los planteamientos teóricos y los desarrollos cuantitativos sobre el tema, y permite obtener resultados robustos y consistentes. La principal limitante del estudio es la falta de mayor información relevante y los datos de corte transversal, ya que se utiliza la segunda encuesta de desarrollo e innovación tecnológica en el sector manufacturero (EDIT II).

Se aporta nueva evidencia al estudio del proceso innovador en Colombia, y los resultados obtenidos constituyen una base para futuras investigaciones sobre innovación al brindar fundamentos teóricos y empíricos a la discusión académica existente sobre los determinantes de la innovación en las firmas; en paralelo se vislumbran las características propias del sector industrial bogotano en cuanto a los procesos de innovación y los determinantes de esta.

En línea con la literatura sobre el tema, los hallazgos indican que el tamaño de la firma, la propiedad extranjera, el capital de conocimiento y el acceso a financiamiento externo son variables relevantes para explicar la inversión en diferentes actividades de innovación tecnológica en el sector manufacturero de la ciudad de Bogotá. También se evidencia una desarticulación de las firmas con los actores del Sistema Nacional de Innovación.

La organización del documento es la siguiente: posterior a esta introducción se analizan los planteamientos teóricos. En la tercera sección se efectúa una revisión

Específicamente se trabaja sobre insumos de la innovación medidos por la inversión en actividades de innovación, a lo que se denomina esfuerzo innovador en este trabajo.

Semestre Económico, volumen 15, №. 32, pp. 129-160 • ISSN 0120-6346, julio-diciembre de 2012, Medellín, Colombia 
de los trabajos empíricos. En la cuarta sección se especifica el modelo. En la quinta sección se analizan los resultados. Para finalizar, se presentan las conclusiones del trabajo, y recomendaciones para una futura agenda de investigación.

\section{TEORÍA DE LA INNOVACIÓN}

\subsection{Teoría de Schumpeter}

¿Qué incentiva las innovaciones? Según Schumpeter (1934) no son los problemas o las necesidades preexistentes los que favorecen las innovaciones, al ser estas las que crean las necesidades que satisfacen. Ya que es el emprendedor el sujeto de la innovación, las viejas industrias fallan al innovar, no por los decrecientes beneficios de la innovación, sino más bien por la débil motivación a innovar. Al respecto Di Maio (2003, p. 14) argumenta que lo que incentiva las innovaciones no es el tamaño potencial de los beneficios, sino la presencia de un emprendedor capaz de hacerlo o la capacidad de la empresa para realizar innovaciones junto con la capacidad de administrar las ventajas y posibilidades para mejorar el desempeño económico.

Estas ideas iniciales de Schumpeter (1934) fueron desarrolladas a comienzos del siglo XX y son notorias en su trabajo, en donde el rol central lo tiene el emprendedor al retar las firmas establecidas mediante la introducción de nuevos bienes y procesos que remplazan los negocios y bienes obsoletos, proceso al cual denominó "destrucción creativa". En este contexto la competencia entre emprendedores y firmas pequeñas es la que favorece la actividad innovadora. A esta conclusión se le conoce como Schumpeter marca I.

No obstante, la noción de competencia de la teoría neoclásica (competencia perfecta entre firmas que producen bienes homogéneos) no sirve como marco de análisis de la innovación en virtud de que las firmas que innovan adquieren cierto grado de poder monopolístico. Morck y Yeung (2001, p. 12) destacan que las firmas que desarrollan formas innovadoras de producción pueden reducir costos y de este modo generar ganancias extra al precio prevaleciente o, de igual manera, al desarrollar nuevos o mejorados productos pueden ganar un exceso de beneficios al ser las únicas empresas que producen el producto.

A partir de aquí, Schumpeter (1942) afirma que la innovación es favorecida por la competencia monopolística, ya que las firmas oligopólicas tienen mayores incentivos a innovar de acuerdo con su posición en el mercado y debido a la capacidad de las grandes corporaciones de explotar la elasticidad de la demanda, atraer científicos y técnicos mejor calificados, y emprender proyectos de investigación y desarrollo (I+D) que firmas pequeñas no estarían en capacidad de realizar. De esta manera 
Determinantes de la innovación: evidencia en el sector manufacturero de Bogotá

se plantea que el tamaño de la firma afecta su disposición a innovar, dado que las empresas grandes tienen la capacidad de usar su poder de mercado para generar recursos extra para destinarlos a la $\mathrm{I}+\mathrm{D}$, lo que da como resultado beneficios extra que recompensan la I+D inicial y favorecen su poder de monopolio. Este conjunto de postulados se conoce como la hipótesis schumpeteriana o Schumpeter marca II.

\subsection{Teoría evolucionista}

El evolucionismo intenta explicar, desde una visión dinámica y endógena, todos los procesos y la evolución que las empresas tienen en el mercado, reconociéndolas como agentes que toman decisiones racionales para evitar que su destino se vea ligado a las condiciones genéticas propias de su aparición; se considera que cada agente se esfuerza para buscar la posición en la cual quiere ubicarse o permanecer.

Con base en esto, la innovación es dirigida por la tecnología que la firma utiliza en la actualidad, la cual depende de las tecnologías usadas en el pasado; por las condiciones de mercado que podrían ser el fundamento de la configuración de la velocidad y la dirección del cambio tecnológico y, por último, por la dirección que tome la tecnología en un momento determinado.

Según Di Maio (2003, p. 19), en la teoría evolucionista la tasa de progreso tecnológico es influenciada por la fuerza que tiene el vínculo entre la tasa de avance científico y el surgimiento de nuevas ideas. Otras influencias radican en el grado de apropiabilidad de las diferentes innovaciones, el patrón de la demanda y las condiciones de mercado.

Sumado a esto en el evolucionismo juega un papel central, en el proceso inno vador, el aprendizaje que se genera a través de la acumulación de experiencia en la producción; por esta razón el aprendizaje es un proceso único e intransferible. Se reconocen tres tipos de aprendizaje: uno es el adquirido en procesos de I+D; el segundo tiene que ver con el diseño y la fabricación, y el último se realiza cuando el producto ha sido usado por el consumidor final.

Además del aprendizaje, es importante destacar el papel que juegan las rutinas en el comportamiento y en el desempeño de la empresa en un sistema, de su estructura organizacional y productiva. Para Nelson y Winter (1982, p.14) el concepto de rutina hace referencia a

Una característica persistente de un organismo determinando su posible comportamiento; son heredables en el sentido en el que los organismos de mañana son generados de los de hoy, tienen las mismas características y son seleccionables en el sentido de que los organismos con ciertas rutinas lo harán mejor que otros, y si es así su importancia relativa aumentará con el tiempo.

Semestre Económico, volumen 15, №. 32, pp. 129-160 • ISSN 0120-6346, julio-diciembre de 2012, Medellín, Colombia 
En los países en desarrollo no es frecuente la búsqueda rutinaria de innovaciones, por lo que la innovación depende en gran medida de las transferencias tecnológicas externas, resultado de las importaciones y las adquisiciones de licencias. La generación de innovación en estos países es producto de la imitación o de la mezcla entre imitar e inventar, a lo que Arocena y Sutz (2001, p.7) denominan la capacidad de innovar en condiciones de escasez.

\subsection{Modelos lineales de innovación: hacia el Sistema Nacional de Innovación}

En el período de la posguerra se emplearon modelos que partían de un enfoque lineal para el análisis de la actividad innovadora; asumían que las nuevas tecnologías y la innovación son el resultado de descubrimientos científicos provenientes de la investigación básica y aplicada (Abrunhosa, 2003; p. 4). Por consiguiente, las políticas tecnológicas estuvieron dirigidas a incentivar la I+D como principal canal para obtener innovaciones. Mansfield (1968) afirma que la tasa de progreso tecnológico depende en gran medida de los recursos destinados por las firmas y el Gobierno a la actividad innovadora. Según Di Maio (2003, p.6), estos dependen de las expectativas de ganancia de los empresarios; de este modo se ve afectada por los componentes de la demanda y la oferta.

En un trabajo pionero, Schmookler (1966) concluye que cambios en los patrones de demanda provenientes del crecimiento del mercado o de la inversión en bienes de capital son más significativos para explicar la innovación que los avances en el estado de conocimiento (demand pull innovation). Esta posición es controvertida por Rosenberg (1974), quien afirma que los esfuerzos innovadores de las firmas son estimulados en mayor medida por los avances científicos. El razonamiento parte de que dichos avances provocan innovaciones a través de nuevos conceptos cuando se incorpora nueva maquinaria o inputs al proceso productivo; a esta hipótesis se le denomino "technology push".

Estos modelos son visiones mecánicas, que explican el proceso de innovación como una relación lineal, sin atender el papel que juegan las instituciones y otros factores económicos sobre la innovación de las firmas. Por consiguiente, se establece que la dinámica innovadora es demasiado compleja, como para ser abordada desde una perspectiva lineal como las propuestas de Schmookler (1966) y Rosenberg (1974).

Las primeras críticas a esos modelos provienen del modelo de cadena de Kline y Rosenberg (1986), que considera la innovación como un proceso complejo, donde las actividades innovadoras son influenciadas por las interacciones entre las firmas, organizaciones del sistema educativo y del sistema tecnológico, mediante feedbacks de información a lo largo de toda la cadena. De acuerdo con Abrunhosa (2003, p. 5) 
Determinantes de la innovación: evidencia en el sector manufacturero de Bogotá

el modelo destaca la importancia de la ciencia, la investigación y del mercado en el proceso innovador, donde se asigna un rol central a la firma y se enfatiza en las interacciones entre las diferentes fases del modelo lineal y el sistema tecnológico.

Conforme a estas ideas, en los últimos años se ha abordado el proceso innovador bajo una perspectiva sistémica, originándose en la década de 1980 el concepto de sistema nacional de innovación (SNI) para explicar las diferencias en el desempeño innovador de los países industrializados (Metcalfe y Ramlogan, 2005, p.5). Los trabajos pioneros (Nelson, 1993; Lundvall, 1992; Freeman, 1995) concluyen que las diferencias en el desempeño tecnológico a través de los Estados radican en las distintas combinaciones de instituciones, así como de las interacciones entre estas.

Dentro de la literatura han surgido distintas definiciones y se le han dado diversos usos al concepto de SIN. Una definición precisa es propuesta por Niosi y otros (1993, p. 212):

El sistema de interacción de empresas privadas y públicas, universidades y agencias gubernamentales, con miras a la producción de la ciencia y la tecnología dentro de las fronteras nacionales. La interacción entre estas unidades puede ser técnica, comercial, jurídico, social y financiero, en la medida en el objetivo de la interacción es el desarrollo, la protección, la financiación o la regulación de la nueva ciencia y la tecnología.

La fortaleza del enfoque SNI para explicar la dinámica innovadora radica en destacar el rol desempeñado por las instituciones y los procesos de mercado. Bajo este enfoque la firma es concebida como una organización de aprendizaje, arraigada dentro de un marco institucional nacional, y por consiguiente, su innovación puede ser impulsada mediante políticas públicas.

El análisis de los anteriores enfoques permite concluir que la innovación no puede ser abordada de manera correcta mediante el uso de esquemas simplificados (modelos lineales); por consiguiente, en la exploración de la dinámica innovadora, además de los factores de mercado (oferta y demanda) y los avances científicos, deben ser consideradas las interacciones entre firmas, universidades, entidades públicas, y demás agentes del SNI.

Para concluir esta sección, se resalta que el proceso de la innovación es un fenómeno complejo en el que prevalecen varias relaciones endógenas y dinámicas, por lo que cada uno de los enfoques teóricos descritos por separado presentan un alcance limitado a la hora de explicar la actividad innovadora; la misma conclusión puede encontrarse en Ruttan (1997), que evalúa el alcance de los avances teóricos realizados en las últimas décadas (cambio tecnológico inducido, teoría evolucionista y Path dependence). 


\section{TRABAJOS EMPÍRICOS SOBRE INNOVACIÓN: REVISIÓN DE LA LITERATURA}

Analizar los determinantes de la actividad innovadora y cuantificar su impacto sobre alguna variable relevante de índole económica (productividad, crecimiento, empleo) ha sido uno de principales objetos de análisis empírico en economía. Estudios empíricos anteriores sobre I+D e innovación como el de Cameron (1998), analizan esta relación mediante una función de producción de conocimiento, en donde se toman como variable explicada los gastos en investigación o patentes. El problema de estos estudios, aparte de los problemas de especificación econométrica, es que usan series agregadas en la estimación, con lo cual se desconoce la diversidad institucional de las firmas, los métodos para realizar I+D y los resultados individuales.

En la actualidad, la mayor disponibilidad de encuestas ha permitido realizar estudios empíricos acerca de los determinantes de la innovación en el ámbito de la firma. Crepón, Duguet y Mairesse (1998) con el objetivo estudiar los vínculos entre productividad, innovación e investigación en Francia utilizan un modelo estructural (conocido como CDM) compuesto por cuatro ecuaciones recursivas: dos para explicar la decisión de la firma de hacer investigación y la intensidad de esta, una para innovación que explica el output innovador en función de la inversión en I+D y la última para la relación innovación-productividad. Los resultados corroboran la correlación positiva existente entre innovación y productividad y los planteamientos de la hipótesis schumpeteriana, al encontrar que la propensión de la firma a invertir en I+D está asociada de forma positiva con el tamaño, la concentración del mercado, el capital humano y los factores tecnológicos y de demanda.

Se ha tomado como marco de referencia el modelo CDM para realizar una gran cantidad de estudios empíricos que encuentran resultados similares en países desarrollados: Griffith y otros (2006) para Francia, Alemania, España y Gran Bretaña, y Mairesse, Marcel y Mohnen (2006) para siete países europeos. En adición a esto, se ha destacado el impacto de la innovación sobre otras variables además de la productividad: Jaumandreu (2003) y Harrison y otros (2008) miden el impacto de las actividades innovadoras sobre el empleo, mediante un modelo que relaciona el crecimiento del empleo con las innovaciones y el crecimiento de las ventas debido a viejos productos y productos innovadores.

Recientemente ha habido un incremento en la producción académica sobre innovación en países en desarrollo: Benavente $(2002,2005)$ para Chile, Chudnovsky, López y Pupato (2004) para Argentina, Romo y Hill de Titto (2006) para México, Cassonni y Rammada (2010) para Uruguay y Crespi y Zúñiga (2010) para seis países latinoamericanos. Entre los principales resultados de esta clase de trabajos se desatacan la importancia del tamaño de la firma y del apoyo público en el proceso innovador; que las firmas multinacionales o expuestas a la competencia internacional 
Determinantes de la innovación: evidencia en el sector manufacturero de Bogotá

son más propensas a innovar, así como escasos vínculos entre empresas y demás agentes del SNI a la hora de innovar.

No obstante, los resultados de algunos de estos trabajos difieren de los encontrados en los países desarrollados. Benavente (2002) y Chudnovsky y otros (2004) no encuentran una correlación positiva entre el output innovador y la productividad. Una razón podría ser que los procesos innovadores en esta clase de países difieren de los realizados en los países desarrollados; de acuerdo con Crespi y Zuñiga (2010, p. 2) desde la teoría se ha demostrado que la imitación y la adquisición de tecnologías son más importantes que la I+D para el aprendizaje y la convergencia.

En Colombia la literatura empírica sobre innovación parte de trabajos descriptivos y estudios de caso; entre estos Durán y otros (2000) caracterizan la innovación en la industria colombiana con los datos de la EDIT I, y Malaver y Vargas (2004) analizan un conjunto de empresas industriales, e identifican que los procesos de innovación son de carácter informal y episódico, producto de bajas inversiones en I+D. Recientemente se han empleado técnicas de análisis cuantitativas más sofisticadas, similares a las utilizadas en los trabajos realizados en los países desarrollados. En esta línea, Langebaek y Vásquez (2007) contrastan las hipótesis neo- Schumpeterianas para el caso colombiano y encuentran que las firmas más grandes, con capital extranjero y mano de obra calificada, realizan un mayor esfuerzo innovador; Vélez (2009) halla los mismos resultados para el caso de las firmas del sector servicios de Bogotá. Entre las limitantes de estos estudios se encuentra que no trabajan algunos aspectos clave del proceso innovador, como el financiamiento de la inversión en innovación o el efecto de las instituciones.

Sin hacer énfasis en el caso colombiano o prestar particular atención a estas variables, ciertos estudios comparativos entre países han encontrado algunos resultados interesantes; Marotta y otros (2007) encuentran que la cooperación con los demás agentes del SNI incrementa la probabilidad de innovar en las firmas de Colombia y Chile. Crespi y Zúñiga (2010) encuentran diversidad en el comportamiento innovador en las firmas de Latinoamérica; como muestra de ello las empresas manufactureras colombianas que recibieron financiamiento público son más innovadoras, a diferencia de las firmas de Uruguay, Argentina y Costa Rica.

\section{MODELO DE DETERMINANTES DE LA INNOVACIÓN}

\subsection{Modelo econométrico}

Dado el problema de la selección de muestra (Heckman, 1979) y la naturaleza estadística de los datos disponibles ${ }^{2}$, para modelar el comportamiento innovador

Véase en Mairesse y Mohnen (2010) un análisis detallado de las características estadísticas de los

Semestre Económico, volumen 15, №. 32, pp. 129-160 • ISSN 0120-6346, julio-diciembre de 2012, Medellín, Colombia 
de la firma se define un modelo Tobit generalizado o tipo II en la clasificación de Amemiya (1985) compuesto por dos ecuaciones: una explica la propensión a innovar y otra la cantidad de recursos invertidos. Sea $i=1, \ldots, n$ un índice de firmas, la ecuación 1 busca explicar la decisión de invertir en innovación a partir de una variable dependiente latente para la firma $i$ tal que:

$$
g_{i}= \begin{cases}1 \text { if } & g_{i}^{*}=X_{1 i} \beta_{0 i}+u_{1 i}>0 \\ 0 \text { if } & g_{i}^{*}=X_{1 i} \beta_{0 i}+u_{1 i} \leq 0\end{cases}
$$

Donde $g_{i}{ }_{i}$ representa algún criterio de decisión, como el valor presente esperado de algún proyecto de inversión; $X_{1 i}^{\prime}$ es el vector que corresponde a la $i$-ésima fila de la matriz $X$ de variables predeterminadas, $\beta_{1 i}$ un vector de parámetros a estimar y $u_{1 i}$ el término error que representa las variables no observadas por sesgo de especificación. Se observa que la firma invierte en actividades de innovación, si $g_{i}$ es positivo o mayor que algún umbral constante, ya sea de naturaleza global o específico a la industria.

Una vez conocida la decisión de inversión de la firma, se procede a explicar la verdadera intensidad del gasto en innovación mediante la ecuación 2:

$$
k_{i}^{*}=X_{2 i} \beta_{2 i}+u_{2 i} \text { if } g_{i}=1
$$

Donde $k_{i}^{*}=k_{i}$, cuando la firma invierte en innovación, i.e. cuando $g_{i}$ es mayor que el umbral mínimo industrial; $X_{2 i}^{\prime}$ es el vector de variables explicativas, $\beta_{2 i}$ un vector de parámetros a estimar y $u_{2 i}$ el término error que resume los determinantes omitidos en el modelo.

De acuerdo con la literatura basada en modelos Tobit generalizados, dado que $k_{i}^{*}$ es observado solo cuando $g_{i}^{*}$ es mayor que el umbral mínimo industrial, se supone una distribución conjunta de los errores para tener un modelo estimable:

$$
\left(\begin{array}{l}
u_{1 i} \\
u_{2 i}
\end{array}\right) \sim N\left[\left(\begin{array}{l}
0 \\
0
\end{array}\right),\left(\begin{array}{cc}
\sigma_{1}^{2} & \rho \sigma_{1} \\
\rho \sigma_{1} & \sigma_{2}^{2}
\end{array}\right)\right]
$$

Donde $N$ denota una distribución normal, $\sigma_{1}$ y $\sigma_{2}$ son los errores estándar de los $u_{1}$ y $u_{2}$ respectivamente y $\rho$ su coeficiente de correlación ${ }^{3}$. La ecua-

datos. Como principal característica se dispone de una muestra truncada y censurada.

3 Este parámetro es crucial, ya que hace que el modelo difiera de un modelo Tobit estándar o un modelo probit. Por consiguiente se probaraá la hipótesis de que este coeficiente es distinto de cero para ver si el modelo de selección es el más adecuado. 
Determinantes de la innovación: evidencia en el sector manufacturero de Bogotá

ción (1) es una ecuación probit, por lo que puede $\sigma_{2}^{2}$ ser normalizada como 1 sin perder generalidad porque la escala de la variable dependiente es no observada.

La estimación del modelo se hace en una misma etapa con el fin de evitar el posible sesgo de selección; para esto se puede emplear el método de máxima verosimilitud o el estimador en dos etapas de Heckman (1979). Se recurre al método de máxima verosimilitud, ya que permite obtener estimadores consistentes y más eficientes que el método de Heckman (1979). Se debe considerar la posible presencia de heteroscedasticidad en los errores, dado que la muestra involucra unidades heterogéneas (empresas de distintos tamaños y sectores), por lo cual en las regresiones se reportan errores estándar robustos.

En este ejercicio se ha establecido una serie de condiciones de exclusión para tener un modelo identificado. En la estimación de la ecuación probit se necesita, al menos, una variable que afecte la decisión de innovar pero no la cantidad de recursos destinados a la innovación, ya que si los regresores de ambas ecuaciones son los mismos, existe la posibilidad de encontrar un alto grado de colinealidad entre las regresiones y el modelo de selección sería inservible.

Para lo anterior se incluyen en la ecuación (1) las variables departamento I+D, bajo el supuesto de que la existencia de un departamento de investigación en el interior de la firma afecte la decisión de innovar, pero no la inversión, más allá de la nómina de los trabajadores, como resaltan Romo y Hill de Titto (2006, p. 35), y la variable inversión rezagada, pues se asume que si una empresa gastó en un año determinado tendrá mayor probabilidad de invertir en el siguiente, debido a la naturaleza plurianual del proceso de innovación. En la ecuación (2) se incluyen las variables de financiamiento (público y privado). No se incluyeron estas variables en la ecuación (1) ya que sería tautológico; una firma que recibe financiamiento para la innovación de manera obvia decidirá invertir.

El resto de variables explicativas que entran en ambas ecuaciones son las mismas. Sin embargo, dadas las restricciones impuestas por el diseño de la EDIT II y el efecto estadístico de quitar observaciones de la muestra, se decidió estimar una versión reducida del modelo que incluye solo las variables de características de la firma y el financiamiento recibido; y una versión extendida que incluye las condiciones de demanda y oferta tecnológica, con las que se busca probar la robustez de los resultados a la inclusión de nuevas variables y determinar si las firmas que tienen vínculos con los agentes del SNI tienden a ser más innovadoras. 


\subsection{Definición de variables}

\subsubsection{Variables dependientes}

- Innova: se crea una variable binaria que toma el valor de 1, si la firma reporta gastos en innovación positivos, o 0 en el caso contrario. Representa la decisión de la firma de involucrarse o no en actividades de innovación.

- Esfuerzo innovador: es el conjunto de actividades desplegadas por las firmas en procura del mejoramiento de su acervo tecnológico. De acuerdo con RICYT (2001, p.38) estas actividades van más allá de la I+D y por esta razón se considera como variable dependiente el logaritmo de la inversión por trabajador en adquisición de tecnologías incorporadas al capital, tecnologías de gestión, tecnologías transversales, capacitación tecnológica, I+D y el conjunto de las anteriores ${ }^{4}$.

\subsubsection{Variables explicativas}

Con base en los planteamientos teóricos y modelos empíricos descritos, se establece que las variables predeterminadas provienen de dos conjuntos: características de la firma y factores externos a esta.

\subsubsection{Características de la firma}

- Tamaño: se aproximará mediante el logaritmo del total del personal empleado por la empresa, con lo que se busca capturar efectos de escala en la decisión de innovar y el gasto en innovación.

- Capital extranjero: la presencia de capital extranjero en la firma permite evaluar el aporte de las compañías multinacionales al desarrollo tecnológico del sector manufacturero local Benavente (2005). Se creó una dummy que toma el valor de 1 si la firma recibe capital extranjero.

- Base de conocimiento existente: que según Vega y otros (2005, p.8) puede ser entendida como "el conjunto de habilidades, conocimientos, y experiencia del que dispone la organización". Puede ser medida en dos dimensiones: la primera es la competencia del capital humano, determinada por el nivel de formación de la fuerza laboral, para lo que se ha construido la variable skills, que es el porcentaje de trabajadores con título universitario (profesional, especialización, maestría, Ph. D.); la segunda dimensión está relacionada con la infraestructura de la firma. Se creó la variable departamento I+D, que toma el valor de 1 si la firma reporta tener un departamento de I+D. Complementario a esto se incluye la variable ideas I+D, si tal departamento es fuente importante de ideas para innovar.

Véase en DANE (2005) para una definición detallada de cada rubro. 
- Inversión rezagada: se incluyen los gastos en innovación en el año 2003 (en logaritmos).

\subsubsection{Factores externos}

- Competencia: en la literatura es común usar medidas de concentración de mercado, como el índice de concentración de Herfindahl para caracterizar la estructura del mercado (competitivo, oligopólico, monopólico). Por desgracia al momento de realizar el estudio no se contó con información sobre ventas de las empresas para la construcción del índice o indicadores semejantes. Por tal razón, del mismo modo que Langebaek y Vásquez (2007) se utilizará el número de empresas pequeñas (menos de 50 trabajadores) sobre el total del sector.

- Condiciones de demanda: son indicadores que se representan mediante dos variables categóricas en una escala de 0 a 3, que miden la importancia otorgada por la firma a si los clientes y la competencia han sido contribuidores de ideas para la innovación en la firma.

Tabla 1. Clasificación de las firmas industriales en la taxonomía de Pavitt

\begin{tabular}{|c|c|}
\hline Taxonomía de Pavitt (1984) & Clasificación CIIU Rev. 3 A.C \\
\hline \multirow{5}{*}{$\begin{array}{l}\text { 1. Intensivos en recursos } \\
\text { naturales }\end{array}$} & 15. Alimentos y bebidas \\
\hline & 16. Tabaco \\
\hline & 20. Madera \\
\hline & 23. Derivados del petróleo \\
\hline & 26. Productos minerales no metálicos \\
\hline \multirow{7}{*}{ 2. productos tradicionales } & 17. Textiles \\
\hline & 18. Prendas de vestir \\
\hline & 19. Productos en cuero \\
\hline & 21. Papel \\
\hline & 22. Impresión y grabaciones \\
\hline & 28. Productos elaborados de metal \\
\hline & 36. Muebles e industrias manufactureras ncp \\
\hline
\end{tabular}




\begin{tabular}{|c|c|}
\hline Taxonomía de Pavitt (1984) & Clasificación CIIU Rev. 3 A.C \\
\hline \multirow{3}{*}{$\begin{array}{l}\text { 3. proveedores especializa- } \\
\text { dos }\end{array}$} & 29. Fabricacion de maquinaria y equipo \\
\hline & 31. Maquinaria y aparatos eléctricos \\
\hline & 33. Instrumentos médicos y de precisión \\
\hline \multirow{4}{*}{$\begin{array}{l}\text { 4. procesos intensivos en } \\
\text { escala }\end{array}$} & $\begin{array}{l}\text { 241. Fabricación de sustancias químicas básicas } \\
\text { 25. Productos de caucho y de plástico }\end{array}$ \\
\hline & 27. Productos metalúrgicos básicos \\
\hline & 34. Vehículos automotores y remolques \\
\hline & 35. Otros tipos de equipos de transporte \\
\hline \multirow{3}{*}{ 5. basados en la ciencia } & 242. Fabricación de otros productos químicos \\
\hline & 30. Maquinaria de oficina, contabilidad e informática \\
\hline & 32. Fabriacion de equipos de comunicación \\
\hline
\end{tabular}

Fuente: elaboración propia con base en Pavitt (1984) y Páscale (2005)

- Condiciones de oferta: se construyeron dos variables categóricas que indican la importancia de la universidad y consultores o expertos como fuentes de ideas para la innovación.

- Financiamiento público: logaritmo del valor financiado por entidades públicas en 2004.

- Financiamiento privado: monto de los créditos otorgados en 2004 (en logaritmos).

- Sector económico: según Pavitt (1984) las diferencias sectoriales en oportunidades tecnológicas, fuentes de la tecnología, exigencias de los clientes las condiciones de apropiabilidad afectan las actividades innovadoras. Para capturar estos efectos se han creado variables dicotómicas de acuerdo con su clasificación de los sectores industriales, adaptándola a la clasificación CIIU, como se muestra en la tabla 1.

\subsection{Estadística descriptiva}

La EDIT II contiene información para un total de 2.469 empresas industriales de Bogotá pero debido a información faltante de algunas variables la muestra final para la estimación está conformada por 2.301 y se toma el año 2004 como período 
Determinantes de la innovación: evidencia en el sector manufacturero de Bogotá

de referencia en virtud de que las cifras empleadas corresponden a este año ${ }^{5}$. Las empresas se dividen entre las que realizaron algún tipo de inversión en innovación y las que no invirtieron; el primer grupo de firmas representa el 76,34\% del total, ajustándose al patrón encontrado por Langebaek y Vásquez (2007, p. 6) para el total de la industria colombiana.

Al analizar la inversión en actividades de innovación en 2004, de acuerdo con la figura 1 se encuentra que el rubro de mayor participación es el de inversión asociada al capital (65\%), seguido de tecnologías de gestión (15\%), mientras que en proyectos de I+D solo se invierte un 3,7\% del total de la inversión. Este hecho indica que la mayoría de lo que se reporta como innovación en el sector manufacturero bogotano corresponde a reposición de activos mediante la adquisición de maquinaria y equipo.

Figura 1. Tipo de inversión

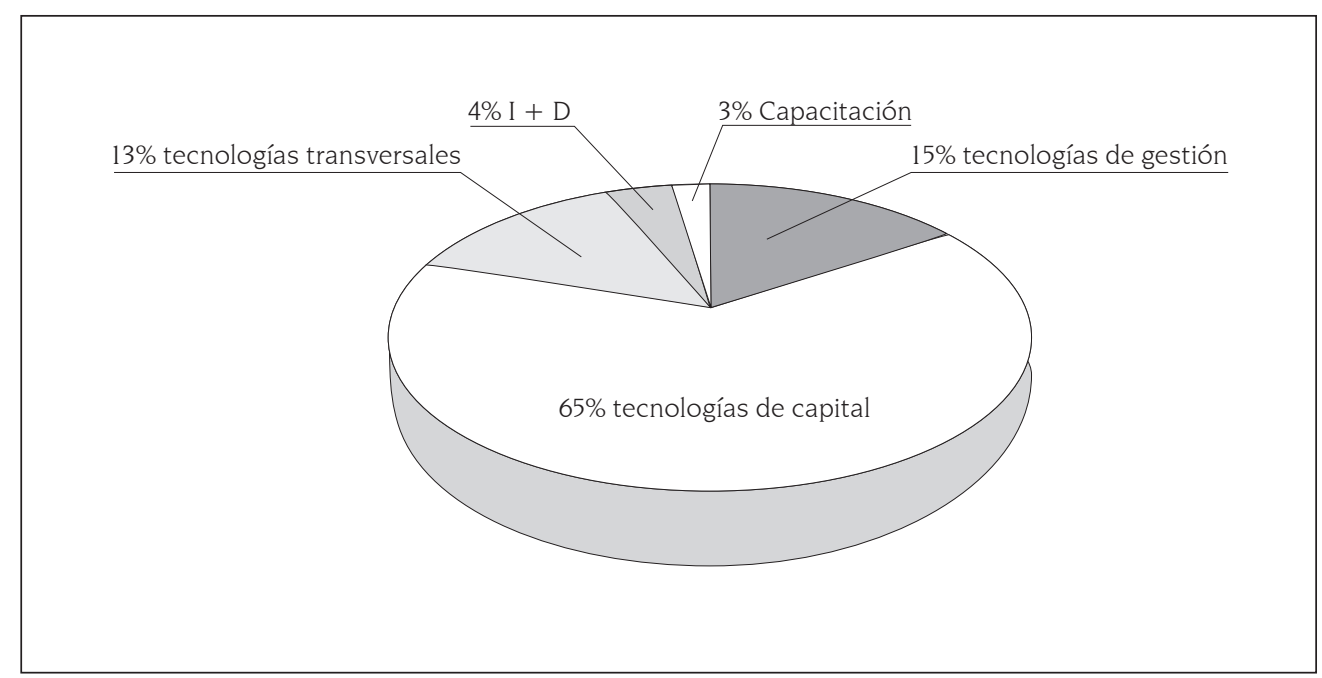

Fuente: elaboración propia. Datos EDIT II

Al concentrar el análisis en los determinantes de la actividad innovadora, un factor relevante es el tamaño de la empresa ${ }^{6}$; si se desagrega de este modo la información se encuentra que las firmas de mayor tamaño son más propensas a realizar actividades de innovación tecnológica; como se observa en la figura 2, mientras que el $44 \%$ y el $70 \%$ de las micro y pequeñas empresas realizó algún tipo de inversión, la proporción en empresas grandes es del 97\%. De aquí que el 82\% de la inversión total de la industria se concentra principalmente en las empresas

${ }_{5}^{5} \quad$ En el anexo A se presentan algunas estadísticas simples para las variables del modelo.

6 Las empresas se clasifican en micro: menos de 10 empleados, pequeñas: de 10 a 50 empleados, medianas: 51 a 200 empleados y grandes de 201 en adelante. 
medianas y grandes, aunque la inversión promedio de las grandes es superior a la de las medianas.

Al observar la dinámica del esfuerzo innovador por parte de las empresas extranjeras ${ }^{7}$ se encuentra que estas son más propensas a innovar. Como se observa en el anexo A el promedio de firmas innovadoras con capital extranjero es mayor al de firmas no innovadoras; se encuentra que el $91 \%$ de firmas con capital extranjero realiza algún tipo de inversión en innovación frente al 67\% de las empresas nacionales.

Figura 2. Propensión a innovar según tamaño

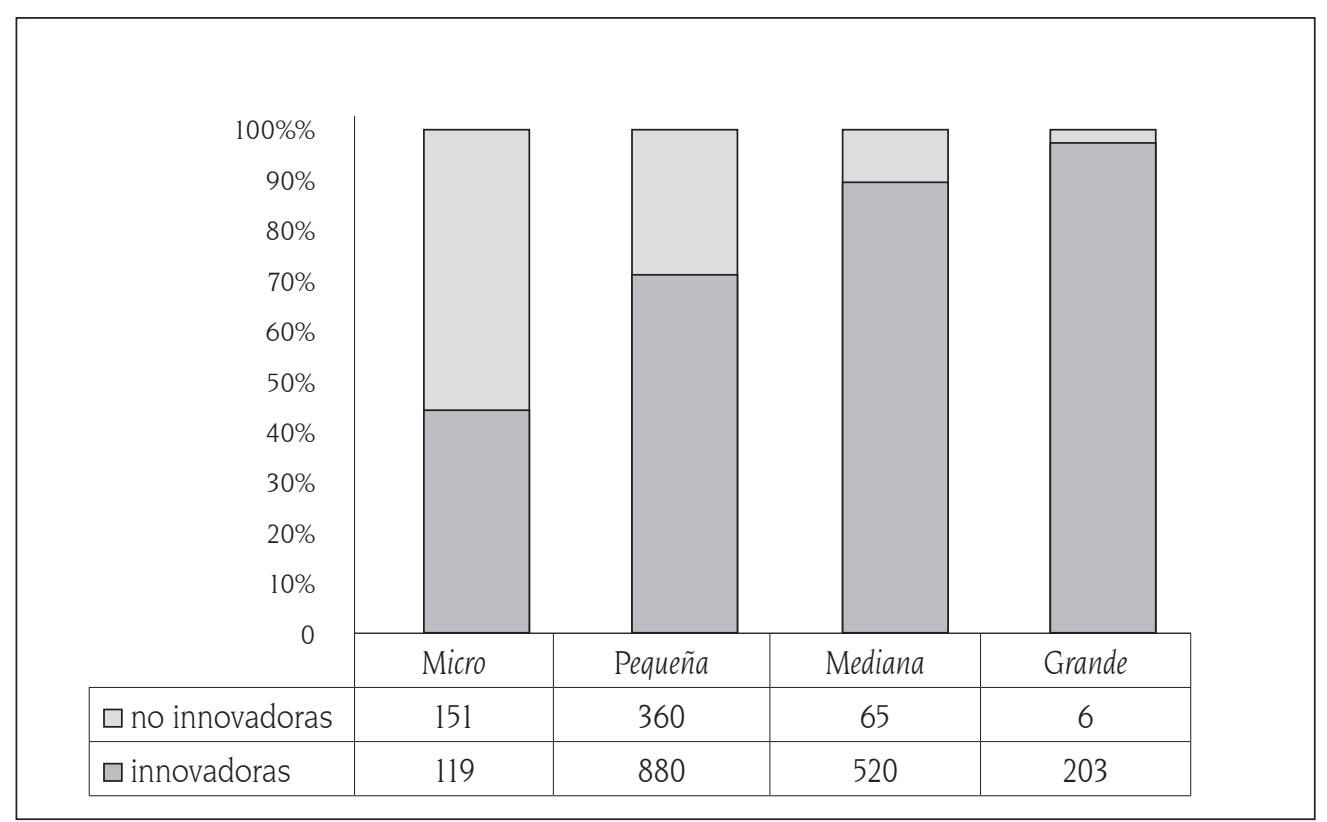

Fuente: elaboración propia. Datos EDIT II

El financiamiento de la inversión es otro factor relevante para la actividad innovadora pues la falta de recursos financieros es uno de los principales obstáculos para la inversión en innovación. Del total de empresas analizadas, solo el 26\% accedió a fuentes externas de financiamiento. Al desagregar estos recursos por fuentes se encuentra que la mayoría (85\%) provienen de préstamos del sector financiero y que solo el 25\% de las empresas recurrieron a las fuentes públicas de financiamiento de la innovación. Así, las empresas industriales bogotanas financian la innovación principalmente con recursos propios, seguido de préstamos bancarios.

\footnotetext{
Se considera una empresa extranjera si más del 25\% de su capital es de origen extranjero.
} 
Por último, como se observa en la figura 3, del total de firmas que realizaron algún tipo de inversión en innovación la mayoría pertenece al sector de productos tradicionales, mientras que solo el 22\% se ubican en los sectores de proveedores especializados y basados en la ciencia, que tienden a ser más propensos a innovar (80\% frente a 70\%). De esta manera se termina con la descripción de los datos para dar paso al análisis de resultados de los modelos econométricos.

Figura 3. Firmas inovadoras por sector industrial

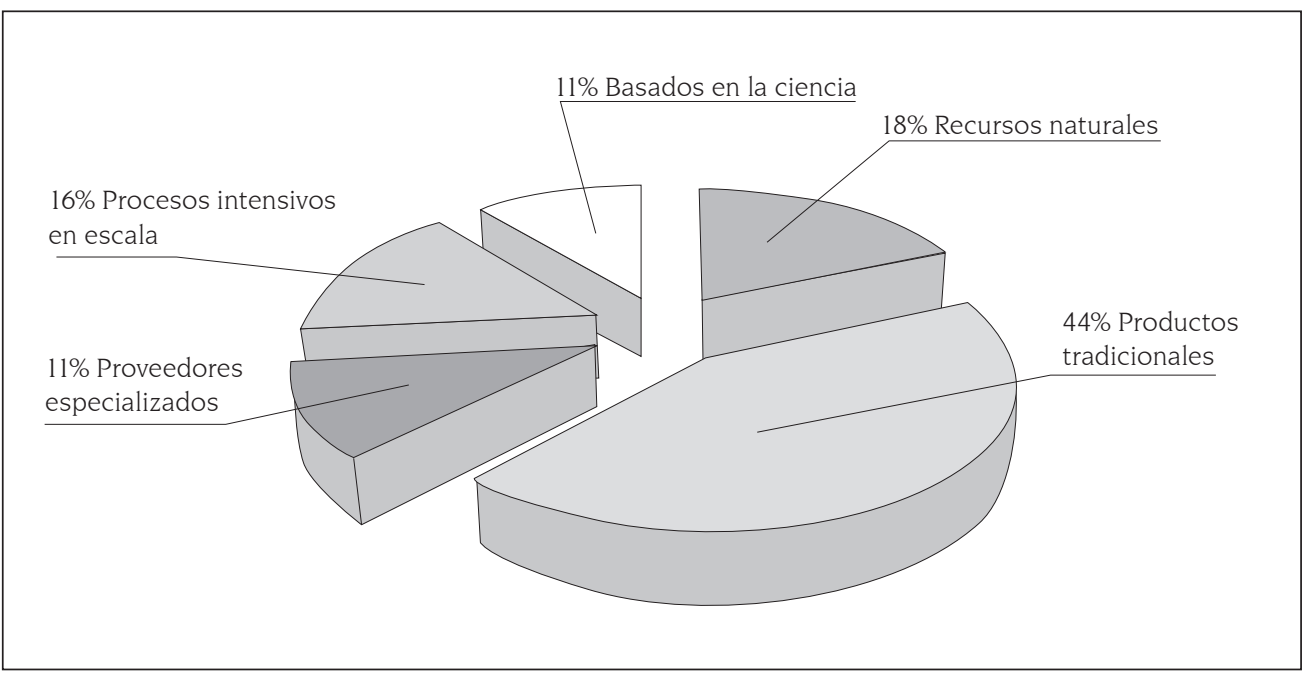

Fuente: elaboración propia. Datos EDIT II

\section{ANÁLISIS DE RESULTADOS}

A continuación se describen los resultados de las estimaciones econométricas del modelo propuesto, de acuerdo con las variables incluidas en los modelos. La tabla 2 presenta los efectos marginales de los modelos probit, y la tabla 3, los resultados de la estimación del esfuerzo innovador en las firmas manufactureras de Bogotá ${ }^{8}$. Los hallazgos permiten establecer que:

- Como era de esperarse las firmas más grandes tienden a ser más innovadoras; la variable tamaño tiene un efecto positivo y estadísticamente significativo en la mayoría de las categorías de gastos de innovación; los efectos marginales más grandes se presentan en las categorías de capital y capacitación $(0.12$ y 0.10 , respectivamente).

\footnotetext{
8 Los resultados de las estimaciones de los modelos extendidos y las pruebas de significancia se encuentran en los anexos B y C.
} 
Este resultado es acorde con el primer planteamiento de la hipótesis schumpeteriana; al respecto Rothwell (1989) sugiere que son varias las ventajas que tienen las empresas grandes sobre las pequeñas a la hora de innovar, entre otras: su capacidad de apalancamiento financiero y diversificación del riesgo, habilidad para atraer personal más capacitado y recursos tecnológicos, su habilidad de ganar economías de escala en I+D, producción y marketing.

Tabla 2. Propensión a innovar en el sector manufacturero de Bogotá 2004

\begin{tabular}{|c|c|c|c|c|c|c|}
\hline Variables & T. Capital & T. Gestión & T. Transversal & IED & Capacitación & Todas \\
\hline \multirow{2}{*}{ Tamaño } & $0,1268^{* * *}$ & $0,0825^{* * *}$ & $0,0545^{* * *}$ & $-0,00084$ & $0,1077^{* * *}$ & $0,0412^{2 * *}$ \\
\hline & $(10,16)$ & $(5,61)$ & $(4,36)$ & $(-0,28)$ & $(8,48)$ & $(5,25)$ \\
\hline \multirow{2}{*}{ Capital ext. } & 0,0004 & 0,0263 & $-0,102^{*}$ & $-0,1023$ & $-0,0835$ & $-0,0302$ \\
\hline & $(0,01)$ & $(0,40)$ & $(-1,86)$ & $(-0,92)$ & $(-1,43)$ & $(-0,72)$ \\
\hline \multirow{2}{*}{ Rivalidad } & $-0,0145$ & $-0,1746$ & $-0,0558$ & 0,0032 & 0,0439 & $-0,1098^{*}$ \\
\hline & $(-0,14)$ & $(-1,29)$ & $(-0,47)$ & $(0,10)$ & $(0,40)$ & $(-1,66)$ \\
\hline \multirow{2}{*}{ Skills } & 0,0013 & $0,0020^{*}$ & $0,0018^{* *}$ & 0,00008 & $0,0035^{* *}$ & 0,00051 \\
\hline & $(1,64)$ & $(1,88)$ & $(2,07)$ & $(0,38)$ & $(3,96)$ & $(0,96)$ \\
\hline \multirow{2}{*}{ Inversión 2003} & $0,0438^{* * *}$ & $0,2466^{* *}$ & $0,0873^{\cdots *}$ & $0,020^{* * *}$ & $0,1109^{\cdots *}$ & $0,0423^{* * *}$ \\
\hline & $(17,53)$ & 23,94 & $(26,53)$ & $(7,79)$ & $(24,71)$ & $(20,01)$ \\
\hline \multirow{2}{*}{ Dpto. I+D } & $-0,0221$ & $-0,0518$ & $-0,0411$ & $0,05274^{* *}$ & 0,0300 & 0,0148 \\
\hline & $(-0,48)$ & $(-0,97)$ & $(-0,79)$ & $(7,41)$ & $(0,55)$ & $(0,51)$ \\
\hline \multirow{2}{*}{ Recursos } & $-0,0438$ & $0,1218^{* * *}$ & $-0,0230$ & $0,032^{* *}$ & 0,0423 & $-0,0113$ \\
\hline & $(-1,36)$ & $(2,96)$ & $(-0,65)$ & $(2,16)$ & $(1,26)$ & $(-0,53)$ \\
\hline \multirow{2}{*}{ Proveedores } & 0,0023 & 0,0401 & 0,0667 & 0,015 & 0,0568 & 0,0163 \\
\hline & $(0,06)$ & $(0,77)$ & $(1,50)$ & $(0,90)$ & $(1,31)$ & $(0,63)$ \\
\hline \multirow{2}{*}{ Escala } & 0,0087 & 0,0388 & $-0,0397$ & 0,0036 & $-0,0180$ & $-0,0126$ \\
\hline & $(0,26)$ & $(0,95)$ & $(-1,11)$ & $(0,36)$ & $(-0,47)$ & $(-0,55)$ \\
\hline \multirow{2}{*}{ Ciencia } & 0,0164 & 0,0112 & $-0,0179$ & $0,042^{*}$ & 0,0613 & 0,0039 \\
\hline & $(0,36)$ & $(0,21)$ & $(-0,36)$ & $(1,93)$ & $(1,38)$ & $(0,14)$ \\
\hline Observaciones & 2301 & 2127 & 2301 & 2301 & 2031 & 2301 \\
\hline wald chi2 & 585,21 & 649,52 & 693,21 & 212,04 & 566,55 & 617,43 \\
\hline$p>$ chi2 & 0,000 & 0,0000 & 0,000 & 0,000 & 0,000 & 0,000 \\
\hline log likelihood & $-1215,2624$ & $-754,09788$ & $-996,71540$ & $-233,9500$ & $-932,5462$ & $-716,02566$ \\
\hline Pseudo R2 & 0,2361 & 0,4805 & 0,3748 & 0,578 & 0,4146 & 0,4484 \\
\hline
\end{tabular}

Los coeficientes reportados son efectos marginales (dy/dx) ${ }^{* * *}$ significativo al $1 \% ;{ }^{* *}$ al $5 \% ;{ }^{*}$ al $10 \%$. estadísticos z entre paréntesis.

Fuente: elaboración propia 
De otro lado, en la ecuación del esfuerzo innovador (tabla 3) se muestra un detalle interesante: la variable tamaño presenta un signo negativo lo cual indicaría un efecto negativo creciente a escala de la inversión en la innovación en las firmas de Bogotá, es decir, que a pesar de que el gasto total aumenta con el tamaño, este incremento es menos que proporcional a medida que la firma crece.

Tabla 3. Esfuerzo innovador en el sector manufacturero de Bogotá 2004

\begin{tabular}{|c|c|c|c|c|c|c|}
\hline Variable & T. Capital & T. Gestión & T. Transversal & IED & Capacitación & Todas \\
\hline \multirow{2}{*}{ Tamaño } & $-0,2712^{* * *}$ & $-0,5417^{* * *}$ & $-0,2653^{* * *}$ & $-0,234^{* *}$ & $-0,2788^{* * *}$ & $-0,0319$ \\
\hline & $(-4,27)$ & $(-9,56)$ & $(-5,41)$ & $(-2,23)$ & $(-5,98)$ & $(-0,74)$ \\
\hline \multirow{2}{*}{ Capital ext. } & $0,6775^{* * *}$ & $0,8397^{* * *}$ & $0,06887^{* * *}$ & 0,433 & $0,6982^{* * *}$ & $0,7552^{* * *}$ \\
\hline & $(4,18)$ & $(4,36)$ & $(4,02)$ & $(1,00)$ & $(5,31)$ & $(5,50)$ \\
\hline \multirow{2}{*}{ Rivalidad } & 0,3444 & 0,0237 & $-0,1914$ & 1,67 & $-0,0928$ & 0,1688 \\
\hline & $(0,75)$ & $(0,04)$ & $(-0,49)$ & $(1,58)$ & $(-0,25)$ & $(0,43)$ \\
\hline \multirow{2}{*}{ Skills } & $0,0220^{* * *}$ & $0,0237^{* * *}$ & $0,0248^{* * *}$ & $0,038^{* * *}$ & $0,0264^{* * *}$ & $0,0297^{* * *}$ \\
\hline & $(5,52)$ & $(5,56)$ & $(7,18)$ & $(4,92)$ & $(8,26)$ & $(9,37)$ \\
\hline \multirow{2}{*}{ F. público } & $0,0365^{* * *}$ & $0,0385^{* * *}$ & $0,0520^{* * *}$ & $0,0735^{* * *}$ & $0,0238^{*}$ & $0,0629^{* * *}$ \\
\hline & $(2,71)$ & $(2,60)$ & $(4,05)$ & $(3,14)$ & $(1,83)$ & $(5,59)$ \\
\hline \multirow{2}{*}{ F. privado } & $0,0742^{* * *}$ & $0,0389^{* * *}$ & $0,0381^{* * *}$ & $-0,0246$ & $0,0239^{* * *}$ & $0,0824^{* * *}$ \\
\hline & $(8,92)$ & $(3,85)$ & $(4,49)$ & $(-1,21)$ & $(3,08)$ & $(11,38)$ \\
\hline \multirow{2}{*}{ Recursos } & $0,4625^{* * *}$ & 0,0179 & $-0,0498$ & 0,5399 & $0,2032^{*}$ & 0,0759 \\
\hline & $(3,50)$ & $(0,11)$ & $(-0,38)$ & $(1,52)$ & $(1,82)$ & $(0,65)$ \\
\hline \multirow{2}{*}{ Proveedores } & $-0,2359$ & $-0,0200$ & $0,2707^{*}$ & $-0,076$ & $0,2564^{*}$ & 0,0230 \\
\hline & $(-1,55)$ & $(-0,12)$ & $(1,91)$ & $(-0,18)$ & $(1,72)$ & $(0,17)$ \\
\hline \multirow{2}{*}{ Escala } & $0,4143^{* * *}$ & $-0,0996$ & 0,0828 & 0,288 & $0,4119^{* * *}$ & $0,3452^{* * *}$ \\
\hline & $(2,90)$ & $(-0,64)$ & $(0,55)$ & $(0,62)$ & $(3,22)$ & $(2,81)$ \\
\hline \multirow{2}{*}{ Ciencia } & $-0,4480^{* * *}$ & $-0,2968^{*}$ & 0,0656 & 0,137 & $0,3029^{* *}$ & $-0,2100$ \\
\hline & $(-2,64)$ & $(-1,67)$ & $(0,41)$ & $(0,40)$ & $(2,22)$ & $(-1,52)$ \\
\hline \multirow{2}{*}{ Constante } & $7,1534^{* * *}$ & $7,8632^{* * *}$ & $6,1568^{* * *}$ & $4,745^{* * *}$ & $4,6139^{* * *}$ & $6,1981^{* * *}$ \\
\hline & $(17,33)$ & $(19,72)$ & $(20,38)$ & $(6,45)$ & $(15,78)$ & $(22,65)$ \\
\hline Observaciones & 2301 & 2127 & 2301 & 2301 & 2031 & 2301 \\
\hline obs. Censuradas & 1083 & 1219 & 1123 & 2151 & 1104 & 579 \\
\hline muestra final & 1218 & 908 & 1178 & 150 & 1197 & 1722 \\
\hline wald chi2 & 177,20 & 162,74 & 153,16 & 52,87 & 231,97 & 316,55 \\
\hline$p>$ chi2 & 0,0000 & 0,0000 & 0,0000 & 0,0000 & 0,0000 & 0,0000 \\
\hline \multirow{2}{*}{$\begin{array}{l}\text { wald test de inde- } \\
\text { pendencia }(\rho=0)\end{array}$} & 32,34 & 76,30 & 68,79 & 15,11 & 22,30 & 56,91 \\
\hline & 0,0000 & 0,0000 & 0,0000 & 0,0000 & 0,0000 & 0,0000 \\
\hline
\end{tabular}

*** Significativo al 1\%; ** al 5\%; ${ }^{*}$ al 10\%.el estadístico del test de independencia es chi2. Estadísticos z entre paréntesis.

Fuente: elaboración propia 
- Tal como se supuso, las firmas que invirtieron en un período anterior tienen mayor probabilidad de ser innovadoras, lo que independientemente de otros factores refleja la persistencia temporal de los gastos en innovación y la dependencia de la trayectoria pasada (path dependence) de los proyectos de innovación. Se encontraron resultados significativos al $1 \%$ en todas las categorías.

- Los resultados para las variables de la base de conocimiento existente permiten corroborar la importancia de la capacidad de absorción, entendida como "la habilidad de la empresa para reconocer el valor de una nueva información externa, asimilarla y aplicarla con fines comerciales" (Cohen y Levinthal, 1990; citado en Vega y otros (2005, p. 1))

La variable skills es significativa en las dimensiones gestión, I+D y tecnologías transversales para explicar la decisión de innovar, y su importancia se revalida en el análisis del esfuerzo innovador, donde es positiva y significativa en todas las categorías. Estos resultados son acordes con la propuesta de la teoría evolucionista de que la capacidad de innovación se da a través de la asimilación, el aprendizaje y la acumulación de conocimiento, si se tiene en cuenta que la contratación del capital humano es una manera de mejorar estos procesos.

Sin embargo, las otras variables empleadas en la base de conocimiento no resultan ser importantes; no existe una relación significativa entre la propensión a innovar y la presencia de un departamento de I+D, con excepción de la inversión en este rubro, ni tampoco las fuentes de ideas provenientes de la I+D son relevantes en las distintas actividades innovadoras de las firmas en promedio. Esta conclusión se corrobora, ya que al censurar la muestra para tomar solo los valores positivos de la inversión, el rubro con menos observaciones es el de I+D. Como se había mencionado las firmas industriales en Bogotá recurren a otras maneras más frecuentes de innovar como la inversión en tecnologías de gestión.

- El acceso al financiamiento incentiva la inversión en innovación; los coeficientes del financiamiento público y del crédito privado son positivos y significativos en todas las categorías. Esto evidencia la presencia de crowding in, es decir, un efecto de complementariedad entre los tipos de financiamiento en lugar de un efecto sustitución.

De aquí se evidencia la importancia de la política pública en materia de financiación, ya que una firma restringida financieramente no puede emprender proyectos innovadores con potencial rentable; por consiguiente, esta ayuda es importante, ya que esta reduce el riesgo financiero de innovar para la firma, vía costos compartidos (Hall y Maffioli, 2008). 
Determinantes de la innovación: evidencia en el sector manufacturero de Bogotá

- La presencia de capital extranjero en la firma resulta ser un factor determinante en todas las actividades innovadoras. El resultado demuestra la importancia de la innovación para las empresas multinacionales, que según Vernon (1966) es la fuerza más importante que subyace a la expansión multinacional.

Es intrigante cinquietante? que la inversión extranjera no afecte la I+D, lo cual podría significar que la transferencia de tecnologías se hace mediante la importación de maquinaria y equipo que traen los avances tecnológicos ya incorporados, lo que Romo y Hill de Titto (2006) denominan embodied technology. Por otra parte, se evidencia también de esta manera el esfuerzo de las firmas por innovar en condiciones de escasez.

- Aun cuando la variable rivalidad no resultó significativa, en la dimensión que reúne el total de la inversión afecta de forma negativa la propensión a innovar, por lo que podrían ser sugestivos los planteamientos de la hipótesis schumpeteriana para el caso analizado. Este resultado debe matizarse en virtud de que la inclusión de este tipo de variable puede representar un problema de endogeneidad, ya que la innovación podría otorgarle cierto grado de poder monopolístico a la firma.

- Por el lado de la demanda, el análisis muestra que la colaboración con los clientes tiene un efecto positivo sobre la mayoría de las actividades innovadoras de la firma. Por el lado de la oferta tecnológica, la opinión de consultores especializados resulta ser más importante a la hora de decidir invertir en innovación; este resultado es significativo en la mayoría de los modelos de inversión. Se establece que ambos factores son importantes para la explicación de la innovación, con lo que se revalidan las tesis de Schmockler (1966) y Rosenberg (1974); sin embargo, no hay una predominancia absoluta de ninguno como se discutía en el marco teórico.

- Ni la competencia ni las universidades resultan ser significativas como fuentes de ideas para innovar. Este resultado se puede comprender a la luz de los hechos estilizados, si se tiene en cuenta que tan solo el 7,2\% de las firmas reportan que estas instituciones son fuentes importantes de ideas para la innovación. Como conclusión se establece que a pesar de las fortalezas que se comentaron sobre el sistema nacional de innovación, en Bogotá resulta preocupante la desarticulación de los agentes que lo componen, por lo que se requerirían medidas de política pública para fortalecerlo.

- Las variables sectoriales que resultaron significativas para la decisión de innovar fueron la de los recursos naturales en los modelos de gestión e I+D, y la variable asociada al sector basado en la ciencia y en la inversión en I+D, lo cual indica 
que estas firmas tienden a innovar más en estos rubros que las del sector de los productos tradicionales (el sector base).

El primer resultado es un indicio de que las firmas cuyos productos son intensivos en el uso de recursos naturales están adaptándose al nuevo paradigma internacional sobre productos y tecnologías más amigables con el medioambiente. El segundo hallazgo era esperado en virtud de lo propuesto por Pavitt (1984): la innovación en el sector basado en la ciencia está relacionada con el surgimiento de nuevos paradigmas tecnológicos a partir de los nuevos avances científicos logrados mediante actividades de I+D.

\section{CONCLUSIONES Y RECOMENDACIONES}

La innovación es considerada como un proceso complejo, que no puede ser correctamente abordada mediante el uso de esquemas simplificados, y de aquí que en la exploración de la dinámica innovadora, además de los factores determinantes tradicionales como las características de la firma, las condiciones de mercado y los avances científicos deben ser consideradas las relaciones externas de las firmas con los demás agentes del Sistema Nacional de Innovación. Por esta razón, el presente artículo trata de contribuir a la literatura al presentar evidencia micro-econométrica sobre cómo estos factores afectan la actividad innovadora de las firmas del sector manufacturero de Bogotá.

Al respecto, una de las principales características de los estudios sobre innovación es que usan modelos uniecuacionales para relacionar alguna medida de la innovación con las características de la firma y la estructura del mercado. Como concluye Benavente (2002), existen severos sesgos que surgen al usar esta clase de modelos, destacándose el de la selectividad. Este problema es enfrentado con el uso de un modelo Tobit generalizado, estimado por máxima verosimilitud.

Los principales resultados arrojados por el modelo indican que la industria manufacturera de Bogotá se ajusta a los patrones de innovación encontrados en otros países en desarrollo; entre los más importantes se destaca que las firmas más grandes tienen mayor propensión a innovar, y una mayor competencia entre empresas afecta de manera negativa la inversión en innovación, por lo que parecen ser sugestivos los planteamientos de la hipótesis schumpeteriana para el caso analizado. Adicionalmente, las empresas multinacionales realizan un mayor esfuerzo innovador y el acceso a financiamiento tiene un efecto complementario (crowding in) sobre la inversión privada en innovación. 
Determinantes de la innovación: evidencia en el sector manufacturero de Bogotá

Amerita resaltar que existen bajos niveles de inversión en I+D: tan solo el 3.7\% del valor invertido por el total de la industria de la ciudad, y que la principal forma de innovación es la inversión en tecnologías incorporadas al capital, lo que es síntoma de que la transferencia de conocimiento desde el extranjero se da mediante la importación de maquinarias y equipos que traen los avances tecnológicos ya incorporados. Lo anterior corrobora la hipótesis de Arocena y Sutz (2001) de innovación en condiciones de escasez en países en desarrollo.

Se encontró que el capital humano parece ser uno de los determinantes más importantes del esfuerzo innovador de las firmas, lo que concuerda con los resultados de los otros trabajos empíricos realizados en Colombia, y la propuesta de la teoría evolucionista según la cual la acumulación y aplicación del conocimiento es un factor determinante de la innovación, como problem solving.

Los principales resultados son robustos a diferentes especificaciones del modelo, esto es, cuando se controla por condiciones de demanda y oportunidades tecnológicas. De esta estimación, se concluye que las firmas que obtienen fuentes de ideas importantes para la innovación de los clientes y consultores especializados son más propensas a emprender proyectos de innovación. Sin embargo, resulta preocupante la desarticulación de las empresas manufactureras de Bogotá con los demás agentes del Sistema Nacional de Innovación, en especial con las universidades, por lo cual se establece que es necesario mejorar los mecanismos existentes para fortalecerlo.

Por otra parte, estudios como el presente deben ser objeto de mejoramiento continuo y actualización, si se quiere tener una mejor comprensión de la innovación en la firma, dado que se afrontan algunas limitaciones; por esta razón se realizan las siguientes recomendaciones para futuros estudios empíricos en Colombia:

- Incluir algunas variables que afectan el proceso innovador de las firmas y que no se tuvieron en cuenta, como el tipo de organización de la firma, la orientación del mercado de la firma y las variables macroeconómicas.

- Se propone evaluar el impacto del financiamiento público sobre los indicadores de insumo y producto innovador de la empresa, tal como se ha hecho en otros países de Latinoamérica. Véanse por ejemplo los trabajos contenidos en Hall y Maffioli (2008).

- Se trató econométricamente solo una parte del total del proceso innovador al nivel de la firma; no se exploró el impacto del esfuerzo innovador sobre el output innovador, ni el efecto de este sobre las variables de desempeño de la firma (productividad, competitividad, entre otras). Por consiguiente, se espera trabajar 
estos temas en futuros estudios cuando se disponga de nueva información sobre las firmas manufactureras de Bogotá y Colombia, para lo cual se pueden utilizar los modelos de Crepón, Duguet y Mairesse (1998) y Jaumandreu (2003).

- La innovación se puede medir por indicadores de producto; no obstante, los de mayor uso (patentes, ventas innovadoras, binarios) presentan ciertos problemas a la hora de evaluar de manera adecuada los resultados del proceso innovador, por lo cual se sugiere construir indicadores que superen estas deficiencias como lo discuten Cassonni y Rammada (2010), quienes proponen un indicador compuesto para medir la innovación.

\section{BIBLIOGRAFÍA}

Abrunhosa, Ana (2003). The national innovation systems approach and the innovation matrix. Paper for the Druid summer conference. Copenhagen. 24p.

Amemiya, Takeshi (1985). Advanced Econometrics. Harvard University Press. Cambridge, MA, 504p.

Arocena, Rodrigo y Sutz, Judith (2001). Revisiting Nelson and Winter from the South: "Learning by Solving" in Underdeveloped Countries. Paper for the DRUID Nelson and Winter Conference, Aalburg, 16p.

Benavente, José (2002). The Role of Research and Innovation in Promoting Productivity in Chile. Documento de Trabajo 200. Departamento de Economía. Universidad de Chile, 29 p.

Benavente, José (2005). Investigación y desarrollo, innovación y productividad: un análisis econométrico a nivel de la firma. En: Estudios de economía, Vol. 32, N. ${ }^{\circ}$, junio, pp. 39- 67.

Cameron, Gavin (1998). Innovation and Growth: a survey of the empirical evidence. Documento, obtenido de: www.nuff.ox.ac.uk/users/cameron/papers/empiric.pdf, el día 20/05/2010. $34 p$.

Cassonni, Adriana y Rammada, Magdalena (2010). Innovation, RED investment and productivity: Uruguayan manufacturing firms. IDB working paper no. 191, 55p.

Chudnovsky, Daniel; Lopez, Andres y Pupato, German (2004). Innovation and Productivity in Developing Countries: A study of Argentine Manufacturing Firms' Behaviour (19922001). Documento de Trabajo 1878, Asociación Argentina de Economía Política, Buenos Aires, 29p.

Cohen, Wesley y Levinthal, Daniel (1990). Absorptive Capacity: A new perspective on learning and innovation. En: Administrative Science Quarterly, N. ${ }^{\circ}$ 35, pp. 128-152. 
Determinantes de la innovación: evidencia en el sector manufacturero de Bogotá

Crepón, Bruno; Duguet, Emmanuel y Mairesse, Jacques (1998). Research, Innovation, and Productivity: An Econometric Analysis at the Firm Level. En: NBER Working Paper, N. ${ }^{\circ}$ 6696, 43p.

Crespi, Gustavo y Zuñiga, Pluvia (2010). Innovation and productivity: evidence from six Latin American countries . En: IDB working paper, N. ${ }^{\circ} 218,38 \mathrm{p}$.

DANE (2005). Segunda Encuesta de Desarrollo e Innovación Tecnológica -EDIT II.

Di Maio, Michele (2003). Explaining Technological Change: A Survey. DRUID Academy Winter 2003, PhD Conference, 26 p.

Duran, Xavier; Ibáñez, Rodrigo; Salazar, Mónica y Vargas, Marisela (2000). La innovación tecnológica en Colombia: características por sector industrial y región geográfica. OcyT, COLCIENCIAS, Bogotá, 198p.

Freeman, Christopher (1995). The National System of Innovation in Historical Perspective. En: Cambridge Journal of Economics, Vol. 19, N. ${ }^{\circ}$ 1, pp. 5-24.

Griffith, Rachel; Huergo, Helena; Mairesse, Jacques y Peters, Bettina (2006). Innovation and Productivity across Four European Countries. En: NBER Working Paper, N.12722, 20p.

Hall, Bronwyn y MaffiolI, Alessandro (2008). Evaluating the Impact of Technology Development Funds in Emerging Economies: Evidence from Latin America. En: NBER Working Paper, N. ${ }^{\circ} 13835,32 \mathrm{p}$.

Heckman, James (1979). Sample selection bias as a specification error. En: Econometrica, N. ${ }^{\circ}$ 47, pp. 153-161.

Jaumandreu, Jordi (2003). Does innovation spur employment. A firm-level analysis using Spanish CIS data, Universidad Carlos III. Madrid. 30p.

Kline, Stephen y Rosenberg, Nathan (1986). An Overview of Innovation. En: R. Landau y N. Rosenberg (eds.). The positive sum strategy.Washington. National Academy Press, 1986, pp. 275-305.

Langebaek, Andrés y Vásquez, Diego (2007). Determinantes de la actividad innovadora en la industria manufacturera colombiana. En: Borradores de Economía, Banco de la República, N. ${ }^{\circ} 433,35 \mathrm{p}$.

Lundvall, Beng-Ake (1992). National Systems of Innovation, Pinter, London, 342p.

Mairesse, Jacques; Marcel, Deganais y Mohnen, Pierre (2006). Innovativity: A comparison Across Seven European Countries. Scientific Series. Centre Interuniversitaire de Recherche en Analyse des Organisations, 22p.

Semestre Económico, volumen 15, №. 32, pp. 129-160 • ISSN 0120-6346, julio-diciembre de 2012, Medellín, Colombia 
Mairesse, Jacques y Mohnen, Pierre (2010). Using Innovations Surveys for Econometric Analysis. En: NBER Working Paper, N. ${ }^{\circ} 15857,40 p$.

Malaver, Florentino y Vargas, Marisela (2004). Los procesos de innovación en la industria colombiana. Resultados de un estudio de casos. En: Cuadernos de Administración, Vol. 12, N. ${ }^{\circ} 28$, pp. 9-51.

Mansfield, Edwin (1968). The Economics of Technological Change. Norton and Company, New York, 257p.

Marotta, Daniela; Michael Mark; Andreas Blom y Kristian Thorn (2007). Human Capital and University-Industry Linkages Role in Fostering Firm Innovation: An Empirical Study of Chile and Colombia. En: The World Bank Policy Research Working Paper, N. ${ }^{\circ} 4443,43 p$.

Metcalfe, Stan y Ramlogan, Ronnie (2005). Innovation systems and the competitive process in developing economies. ESRC centre for research on innovation and competition, Sao Paulo,13p.

Morck, Randall y Yeung, Bernard (2001). The economic determinants of innovation, Industry Canada research paper program. En: Occasional paper, N. ${ }^{\circ}$ 25, 94p.

Nelson, Richard y Winter, Sidney (1982). An evolutionary theory of economic change. Cambridge, Mass: Harvard University Press, 437p.

Nelson, Richard (ed.) (1993). National Innovation Systems: A Comparative Analysis. Oxford University Press, junio 3, 560p.

Niosi, Jorge; Michael, Crow; Bertrand, Bellon y Saviotti, Paolo (1993). National Systems of Innovation: In Search of a Workable Concept. En: Technology in Society, Vol. 15, N.. 2, pp. 207-227.

Páscale, Ricardo (2005). Gestión del conocimiento, innovación y productividad. Exploración del caso de la industria manufacturera uruguaya. Tesis doctoral sobre la Sociedad de la Información y el Conocimiento, 148p.

Pavitt, Keith (1984). Sectorial Patterns of technical change: Towards a taxonomy and a Theory. En: Research Policy, N. ${ }^{\circ}$ 13, pp. 343-373.

RICYT, (2001). Manual de Bogotá. En línea. COLCIENCIAS/OCYT. 102p. http://www.uis. unesco.org/Library/Documents/Bogota\%20Manual_Spa.pdf. Consultado el 17/10/12.

Romo, David y Hill De Titto, Pablo (2006). Los determinantes de las Actividades Tecnológicas en México. Documentos de Trabajo en Ciencia y Tecnología. CIDECYT, 63p. 
Determinantes de la innovación: evidencia en el sector manufacturero de Bogotá

Rosenberg, Nathan (1974). Science, innovation and economic growth. En: Economic Journal, N. ${ }^{\circ} 84$, pp. $90-108$.

Rothwell, Roy (1989). Small firms, innovation and industrial change. En: Small Business Economics, $\mathrm{N}^{\circ} 1$, pp. 51-64.

Ruttan, Vernon (1997). Induced Innovation, Evolutionary Theory and Path Dependence: Sources of Technical Change. En: Economic Journal, vol. 107, pp. 1520-1529.

Schmookler, Jacob (1966). Invention and Economic Growth. la ed., Harvard University Press, Cambridge, 348p.

Schumpeter J. (1934). The Theory of Economic Development: An Inquiry into Profits, Capital, Credit, Interest, and the Business Cycle. Harvard University Press, Cambridge, MA, 255p.

Schumpeter, Joseph. (1942). Capitalismo, Socialismo, y Democracia. Ediciones orbis, Orbis 1983, Barcelona, 512p.

Vega, Jaider; Fernandez, Ignacio; Gutiérrez, Antonio y Manjarres, Liney (2005). Los Determinantes de la Innovación Tecnológica en la Empresa: Una Aproximación A Través del Concepto de Capacidad de Absorción. XI Seminario Latino-Iberoamericano de Gestión Tecnológica, 18p.

Vernon, Raymond (1966).International investment and international trade in the product cycle. En: Quarterly Journal of Economics, N. ${ }^{\circ} 80$, pp. 190-207.

Vélez, Jorge (2009). Determinantes de la inversión en innovación en el sector servicios de Bogotá: estimaciones econométricas a nivel de la firma. Trabajo de Grado para optar al título de Economista. Universidad de la Salle, Bogotá, 64 p. 


\section{ANEXOS}

ANEXO A.

ESTADÍSTICAS DESCRIPTIVAS

\begin{tabular}{|l|c|c|c|c|c|c|}
\cline { 2 - 8 } \multicolumn{1}{c|}{} & \multicolumn{2}{c|}{ Muestra completa } & \multicolumn{2}{c|}{ Innovadoras } & \multicolumn{2}{c|}{ No innovadora } \\
\hline Variable & Media & Desv.Estd. & Media & Desv. Estd. & Media & Desv.Estd. \\
\hline Tamaño & 88.637 & $(257.58)$ & 108.70 & $(293.93)$ & 29.257 & $(48.483)$ \\
\hline Capital Ext. & 0.090 & $(0.286)$ & 0.108 & $(0.311)$ & 0.030 & $(0.172)$ \\
\hline Rivalidad & 0.466 & $(0.120)$ & 0.464 & $(0.120)$ & 0.475 & $(0.120)$ \\
\hline Skills & 15.953 & $(15.549)$ & 16.490 & $(15.161)$ & 14.356 & $(16.545)$ \\
\hline Inversión 2003* & 6.578 & $(5.412)$ & 8.309 & $(4.790)$ & 0.993 & $(2.985)$ \\
\hline Dpto. I+D & 0.103 & $(0.304)$ & 0.125 & $(0.331)$ & 0,039 & $(0,194)$ \\
\hline Recursos & 0.185 & $(0.388)$ & 0.188 & $(0.391)$ & 0,174 & $(0,379)$ \\
\hline Tradicionales & 0.441 & $(0.496)$ & 0.440 & $(0.496)$ & 0,443 & $(0,497)$ \\
\hline Proveedores & 0.104 & $(0.305)$ & 0.108 & $(0.311)$ & 0,089 & $(0,285)$ \\
\hline Escala & 0.157 & $(0.364)$ & 0.155 & $(0.362)$ & 0,166 & $(0,372)$ \\
\hline Ciencia & 0.094 & $(0.292)$ & 0.106 & $(0.308)$ & 0,056 & $(0,231)$ \\
\hline Fin. Privado* & 2.429 & $(4.781)$ & 2.993 & $(5,153)$ & 0,611 & $(2,571)$ \\
\hline Fin. Público* & 0.692 & $(2.726)$ & 0.895 & $(3,071)$ & 0,038 & $(0.662)$ \\
\hline Clientes & 0.989 & $(1.322)$ & 1,024 & $(1,332)$ & 0,427 & $(0.996)$ \\
\hline Competencia & 0.563 & $(1.068)$ & 0.586 & $(1,084)$ & 0,194 & $(0.657)$ \\
\hline Universidad & 0.256 & $(0.742)$ & 0.267 & $(0.758)$ & 0,067 & $(0.350)$ \\
\hline Consultores & 0.451 & $(0.983)$ & 0.470 & $(1,001)$ & 0,145 & $(0.567)$ \\
\hline ideas I+D & 0.153 & $(0.360)$ & 0.160 & $(0.367)$ & 0,038 & $(0.194)$ \\
\hline Observaciones & & 2304 & & 1722 & & 582 \\
\hline
\end{tabular}

* Variables en logaritmos. "Innovadoras" se refiere al grupo de empresas que realizo algún tipo de inversión en innovación.

Fuente: elaboración propia 
Determinantes de la innovación: evidencia en el sector manufacturero de Bogotá

ANEXO B.

PROPENSIÓN A INNOVAR EN EL SECTOR MANUFACTURERO DE BOGOTÁ 2004 (MODELO EXTENDIDO)

\begin{tabular}{|c|c|c|c|c|c|c|}
\hline Variables & T. Capital & T. Gestión & T. Transversal & IED & Capacitación & Todas \\
\hline \multirow{2}{*}{ Tamaño } & $0,0872^{* * *}$ & $0,0461^{* * *}$ & 0,0016 & $-0,0056$ & $0,0631^{* * *}$ & $0,0154^{* * *}$ \\
\hline & $(7,16)$ & $(2,95)$ & $(0,14)$ & $(-1,37)$ & $(5,32)$ & $(3,05)$ \\
\hline \multirow{2}{*}{ Capital ext. } & 0,0458 & 0,0225 & $-0,0592$ & $-0,0082$ & $-0,0534$ & - \\
\hline & $(1,05)$ & $(0,36)$ & $(-1,17)$ & $(-0,58)$ & $(-1,08)$ & -- \\
\hline \multirow{2}{*}{ Rivalidad } & $-0,0021$ & $-0,2106$ & $-0,0559$ & 0,01 & 0,0477 & $-0,0668$ \\
\hline & $(-0,02)$ & $(-1,48)$ & $(-0,47)$ & $(0,28)$ & $(0,47)$ & $(-1,65)$ \\
\hline \multirow{2}{*}{ Skills } & 0,0074 & 0,0018 & $0,0016^{*}$ & 0,000021 & $0,0028^{* * *}$ & 0,0002 \\
\hline & $(0,85)$ & $(1,47)$ & $(1,73)$ & $(0,07)$ & $(3,17)$ & $(0,78)$ \\
\hline \multirow{2}{*}{ Dpto. I+D } & $-0,0199$ & $-0,0321$ & $-0,0625$ & $0,0636^{* *}$ & 0,0164 & $-0,0024$ \\
\hline & $(-0,45)$ & $(-0,55)$ & $(-1,30)$ & $(2,10)$ & $(0,34)$ & $(-0,12)$ \\
\hline \multirow{2}{*}{ Cliente } & $-0,0012$ & $0,0298^{* *}$ & 0,0133 & $0,0101^{* * *}$ & $-0,0031$ & $0,0076^{*}$ \\
\hline & $(-0,13)$ & $(2,32)$ & $(1,26)$ & $(3,21)$ & $(-0,32)$ & $(1,65)$ \\
\hline \multirow{2}{*}{ Competencia } & 0,0078 & 0,0206 & $0,0263^{*}$ & 0,0047 & 0,0169 & 0,0076 \\
\hline & $(0,64)$ & $(1,23)$ & $(1,89)$ & $(1,22)$ & $(1,52)$ & $(1,21)$ \\
\hline \multirow{2}{*}{ Universidad } & $-0,0277$ & $-0,0070$ & 0,0072 & 0,0062 & $-0,0051$ & 0,0015 \\
\hline & $(-1,56)$ & $(-0,26)$ & $(0,35)$ & $(1,25)$ & $(-0,25)$ & $(0,17)$ \\
\hline \multirow{2}{*}{ Consultores } & $0,0342^{* *}$ & $0,0450^{* *}$ & $0,0258^{*}$ & $-0,0027$ & $0,0326^{* *}$ & 0,0036 \\
\hline & $(2,38)$ & $(2,32)$ & $(1,76)$ & $(-0,58)$ & $(2,11)$ & $(0,52)$ \\
\hline \multirow{2}{*}{ Ideas I+D } & $-0,0042$ & - & 0,0528 & $-0,0038$ & $-0,0060$ & 0,0129 \\
\hline & $(-0,11)$ & - & $(1,31)$ & $(-0,35)$ & $(-0,14)$ & $(0,78)$ \\
\hline \multirow{2}{*}{ Recursos } & $-0,0399$ & $0,1377^{* * *}$ & $-0,0329^{* *}$ & 0,0499 & $0,0701^{* *}$ & 0,0110 \\
\hline & $(-1,20)$ & $(3,45)$ & $(-0,93)$ & $(-2,41)$ & $(2,47)$ & $(0,92)$ \\
\hline \multirow{2}{*}{ Proveedores } & $-0,0254$ & 0,0401 & 0,0404 & 0,0189 & 0,0300 & 0,0106 \\
\hline & $(-0,64)$ & $(0,78)$ & $(0,98)$ & $-0,92$ & $(0,78)$ & $(0,80)$ \\
\hline \multirow{2}{*}{ Escala } & 0,0074 & 0,0363 & $-0,0480$ & $-0,0016$ & $-0,0186$ & $-0,0130$ \\
\hline & $(0,22)$ & $(0,88)$ & $(-1,37)$ & $(-0,13)$ & $(-0,54)$ & $(-0,90)$ \\
\hline
\end{tabular}


Esteban Álvarez • William Garć́a

\begin{tabular}{|l|c|c|c|c|c|c|}
\hline \multicolumn{1}{|c|}{ Variables } & T. Capital & T. Gestión & T. Transversal & IED & Capacitación & Todas \\
\hline \multirow{2}{*}{ Ciencia } & $-0,0139$ & $-0,0027$ & $-0,0530$ & $0,0548^{*}$ & 0,0521 & 0,0082 \\
\cline { 2 - 7 } & $(-0,32)$ & $(-0,05)$ & $(-1,10)$ & $(1,88)$ & $(1,39)$ & $(0,51)$ \\
\hline \multirow{2}{*}{ Inversión 2003 } & $0,0233^{* * *}$ & $0,0819^{* * *}$ & $0,0603^{* * *}$ & $0,0221^{* * *}$ & $0,0772^{* * *}$ & $0,0068^{* * *}$ \\
\cline { 2 - 7 } & $(10,15)$ & $(23,23)$ & $(22,12)$ & $(6,98)$ & $(23,77)$ & $(6,25)$ \\
\hline Observaciones & 1761 & 1639 & 1761 & 1761 & 1761 & 1572 \\
\hline Wald chi2 & 262,74 & 495,38 & 456,76 & 212.76 & 437,48 & 96,46 \\
\hline p > chi2 & 0,0000 & 0,0000 & 0,0000 & 0,0000 & 0,0000 & 0,0000 \\
\hline log likelihood & $-955,54565$ & $-646,51838$ & $-794,95932$ & $-204,63314$ & $-722,4433$ & $-321,69587$ \\
\hline Pseudo R2 & 0,1329 & 0,4271 & 0,2955 & 0,5954 & 0,3679 & 0,1540 \\
\hline
\end{tabular}

Los coeficientes reportados son efectos marginales (dy/dx)** significativo al 1\% ; ** al 5\%; ${ }^{*}$ al $10 \%$. estadísticas z entre paréntesis

Fuente: elaboración propia 
Determinantes de la innovación: evidencia en el sector manufacturero de Bogotá

ANEXO C.

ESFUERZO INNOVADOR EN EL SECTOR MANUFACTURERO DE BOGOTÁ 2004 (MODELO EXTENDIDO)

\begin{tabular}{|c|c|c|c|c|c|c|}
\hline Variable & T. Capital & T. Gestión & T. Transversal & IED & Capacitación & Todas \\
\hline \multirow{2}{*}{ Tamaño } & $-0,2742^{* * *}$ & $-0,5541^{* * *}$ & $-0,2911^{* * *}$ & $-0,3433^{* * *}$ & $-0,3066^{* * *}$ & $-0,0566$ \\
\hline & $(-4,81)$ & $(-10,29)$ & $(-6,22)$ & $(-3,44)$ & $(-6,59)$ & $(-1,35)$ \\
\hline \multirow{2}{*}{ Capital ext. } & $0,6373^{* * *}$ & $0,8484^{* * *}$ & $0,6643^{* * *}$ & 0,3657 & $0,7304^{* * *}$ & $0,7241^{* * *}$ \\
\hline & $(3,89)$ & $(4,49)$ & $(3,93)$ & $(0,90)$ & $(5,46)$ & $(5,66)$ \\
\hline \multirow{2}{*}{ Rivalidad } & 0,1486 & $-0,1949$ & $-0,4229$ & 0,8021 & $-0,2316$ & 0,1524 \\
\hline & $(0,32)$ & $(-0,35)$ & $(-1,10)$ & $(0,82)$ & $(-0,61)$ & $(0,40)$ \\
\hline \multirow{2}{*}{ Skills } & $0,0219^{* * *}$ & $0,0209^{* * *}$ & $0,0217^{* * *}$ & $0,0313^{* * *}$ & $0,0249^{* * *}$ & $0,0284^{* * *}$ \\
\hline & $(5,30)$ & $(4,98)$ & $(6,28)$ & $(4,34)$ & $(7,65)$ & $(8,91)$ \\
\hline \multirow{2}{*}{ F Público } & $0,0332^{* *}$ & $0,0359^{* s}$ & $0,0466^{* * *}$ & $0,0701^{* * *}$ & 0,0207 & $0,0551^{* * *}$ \\
\hline & $(2,40)$ & $(2,48)$ & $(3,65)$ & $(3,06)$ & $(1,57)$ & $(4,99)$ \\
\hline \multirow{2}{*}{ F Privado } & $0,0749^{* * *}$ & $0,0392^{* * *}$ & $0,0358^{* * *}$ & $-0,0208$ & $0,0208^{* * *}$ & $0,0736^{* * *}$ \\
\hline & $(8,92)$ & $(3,99)$ & $(4,24)$ & $(-1,06)$ & $(2,71)$ & $(10,30)$ \\
\hline \multirow{2}{*}{ Cliente } & 0,0071 & 0,0224 & $-0,0077$ & $-0,2170^{* *}$ & 0,0591 & $0,0867^{* *}$ \\
\hline & $(0,16)$ & $(0,51)$ & $(-0,21)$ & $(-2,08)$ & $(1,60)$ & $(2,56)$ \\
\hline \multirow{2}{*}{ Competencia } & $-0,0876^{*}$ & $-0,0375$ & 0,0435 & $-0,0483$ & $-0,0306$ & $-0,0426$ \\
\hline & $(-1,76)$ & $(-0,77)$ & $(1,00)$ & $(-0,51)$ & $(-0,70)$ & $(-1,13)$ \\
\hline \multirow{2}{*}{ Universidad } & 0,0016 & $-0,073$ & $-0,0224$ & $-0,1023$ & 0,0212 & $-0,0532$ \\
\hline & $(-0,02)$ & $(-1,02)$ & $(-0,39)$ & $(-0,84)$ & $(0,37)$ & $(-1,08)$ \\
\hline \multirow{2}{*}{ Consultores } & $-0,0273$ & $0,1333^{* *}$ & $0,1134^{* *}$ & $-0,2140^{* *}$ & 0,0515 & $0,1551^{* * *}$ \\
\hline & $(-0,52)$ & $(2,41)$ & $(2,35)$ & $(1,97)$ & $(1,09)$ & $(4,01)$ \\
\hline \multirow{2}{*}{ Ideas I+D } & 0,1737 & - & $0,2640^{* *}$ & 0,2656 & 0,0985 & $0,2102^{*}$ \\
\hline & $(1,22)$ & - & $(1,99)$ & $(1,03)$ & $(0,83)$ & $(1,92)$ \\
\hline \multirow{2}{*}{ Recursos } & $0,4643^{* * *}$ & $-0,0970$ & $-0,0972$ & 0,1102 & 0,1436 & 0,0591 \\
\hline & $(3,39)$ & $(-0,62)$ & $(-0,73)$ & $(0,31)$ & $(1,24)$ & $(0,52)$ \\
\hline \multirow{2}{*}{ Proveedores } & $-0,1711$ & $-0,0174$ & $0,2818^{* *}$ & $-0,2441$ & 0,2313 & 0,0182 \\
\hline & $(-1,08)$ & $(-0,10)$ & $(1,98)$ & $(-0,63)$ & $(1,51)$ & $(0,14)$ \\
\hline
\end{tabular}


Esteban Álvarez • William Garća

\begin{tabular}{|l|c|c|c|c|c|c|}
\hline \multicolumn{1}{|c|}{ Variable } & T. Capital & T. Gestión & T. Transversal & IED & Capacitación & Todas \\
\hline \multirow{2}{*}{ Escala } & $0,3997^{* * *}$ & $-0,1116$ & 0,0814 & 0,1072 & $0,2867^{* *}$ & $0,2971^{* *}$ \\
\cline { 2 - 7 } & $(2,69)$ & $(-0,73)$ & $(0,53)$ & $(0,22)$ & $(2,27)$ & $(2,40)$ \\
\hline \multirow{2}{*}{ Ciencia } & $-0,4199^{* *}$ & $-0,2502$ & 0,0267 & $-0,1548$ & 0,2214 & $-0,2758^{* *}$ \\
\cline { 2 - 7 } & $(-2,40)$ & $(-1,38)$ & $(0,17)$ & $(-0,46)$ & $(1,57)$ & $(-1,98)$ \\
\hline \multirow{2}{*}{ Constante } & $7,3248^{* * *}$ & $8,0113^{* * *}$ & $6,3361^{* * *}$ & $6,1678^{* * *}$ & $4,7925^{* * *}$ & $6,2393^{* * *}$ \\
\cline { 2 - 7 } & $(19,70)$ & $(21,62)$ & $(22,53)$ & $(8,85)$ & $(15,98)$ & $(23,90)$ \\
\hline Observaciones & 1761 & 1639 & 1761 & 1761 & 1761 & 1761 \\
\hline obs. Censuradas & 561 & 741 & 598 & 1614 & 621 & 103 \\
\hline muestra final & 1200 & 898 & 1163 & 147 & 1140 & 1658 \\
\hline wald chi2 & 177,82 & 166,09 & 165,44 & 64,80 & 238,08 & 377,67 \\
\hline p >chi2 & 0,0000 & 0,0000 & 0,0000 & 0,0000 & 0,0000 & 0,0000 \\
\hline \multirow{2}{*}{$\begin{array}{l}\text { wald test de indepen- } \\
\text { dencia }(\rho=0)\end{array}$} & 32,22 & 71,52 & 68,33 & 12,29 & 25,04 & 24,66 \\
\cline { 2 - 7 } & 0,0000 & 0,0000 & 0,0000 & 0,0005 & 0,0000 & 0,0000 \\
\hline
\end{tabular}

*** significativo al $1 \% ;{ }^{* *}$ al 5\%; ${ }^{*}$ al $10 \%$ estadísticos z entre paréntesis

Fuente: elaboración propia 